\title{
FINITE DEFORMATIONS OF FIBRE-REINFORCED ELASTIC SOLIDS WITH FIBRE-BENDING STIFFNESS - PART II: DETERMINATION OF THE SPHERICAL PART OF THE COUPLE-STRESS
}

\author{
K.P. Soldatos \\ School of Mathematical Sciences, University of Nottingham, Nottingham NG7 2RD, UK \\ Email Address: kostas.soldatos@nottingham.ac.uk
}

\begin{abstract}
The indeterminacy of the spherical part of the couple-stress is a well-known drawback of any theoretical formulation stemming from the Cosserat couple-stress theory of elasticity. The relevant theory of finite elastic deformations of solids reinforced by a family of fibres that resist bending [1] is not an exception. The present communication extends and completes that theory in a manner that enables it to measure the spherical part of the couple-stress tensor outside the conventional equilibrium considerations. To achieve this, the present study reconsiders an extra piece of information that has surprisingly emerged already in [1] but, so far, is left unexplained and unexploited. Namely, the fact that the energy stored in a fibrous composite elastic solid with fibre-bending stiffness involves a couple-stress generated term which does not influence the relevant couple-stress constitutive equation. The thus resulting new theoretical development complements the theory presented in [1] without dismissing any of the theoretical results detailed or the conclusions drawn there. Its validity embraces boundary value problems concerning both finite and infinitesimal elastic deformations of polar fibrous composites. In the latter case, its applicability is also tested and verified, through the successful determination of the spherical couple-stress of a polar transversely isotropic elastic plate subjected to pure bending.
\end{abstract}

Keywords: Couple-stress theory, Elasticity, Fibre-reinforced solids, Fibres resistant in bending, Generalised Cosserat theory, Polar elasticity, Spherical part of the couple-stress.

\section{Introduction}

The theory of elastic solids reinforced by a family of fibres resistant in bending [1] is a Cosserattype [2] couple-stress theory and, as such, considers that the stress field is non-symmetric. The theory [1] is mainly concerned with large elastic deformations and is, therefore, nonlinear. Nevertheless, its linearised version (see section 9 of [1] as well as [3]) is directly applicable in the regime of infinitesimal deformations and can therefore be felt more relevant to the actual Cosserat formulation [2] which is also linear. By now, both the linear and non-linear versions of the theory presented in [1] are well established, though, still, both versions involve a couple of interesting features that require further consideration and study.

The first of those features is a well-known, essentially standard drawback of Cosserat-type formulations according to which the resulting constitutive equations leave the spherical part of the couple-stress indeterminate (e.g. [4-13]). That drawback is a consequence of the fact that all Cosserat-type formulations, including [1], begin by postulating that the couple-stress field is 
energetically reciprocal to the gradient of the general rotation field of the deformation. This postulation may be regarded as trivially valid in cases of material isotropy. However, it can be misleading in cases of material anisotropy that is due to fibre presence or, more generally, to the presence of some preference material direction, where the rotation field of the latter may differ considerably from its general deformation counterpart. In such cases, there is generally no information available to guide the selection of the dominant rotation field, namely a rotation field whose gradient is indeed energetically reciprocal to the couple-stress field.

Still though, the theory developed in [1] leaves available a relevant, extra piece of information that has not been taken adequately into consideration, mainly because its source is not yet fully understood. This, second feature of interest, stems from the observation that the energy stored in a fibrous elastic solid with fibre-bending stiffness involves a couple-stress related term which, surprisingly, does not feed into the couple-stress constitutive equation. The emergence of that extra energy term, then, creates a feelings of relevance and correlation with the spherical part of the couple-stress (in brief spherical couple-stress), which also leaves the constitutive equations unaffected.

A connection mechanism between the implied extra energy term and the spherical couplestress thus has recently been proposed [14] and, subsequently, applied successfully on a simple, linear elasticity, boundary value problem of plate bending [15]. That mechanism proposed a modification of the widely used, linear Cosserat theory [2] that enables replacement of the standard, displacement-based rotation/spin field with some alternative rotation/spin field that may be felt more dominant in specific classes of boundary value problems. Its subsequent application in the special case of unidirectional fibrous composites $[14,15]$ succeeded to provide an estimate of the spherical couple-stress by (i) selecting the gradient of the fibre-spin vector as energetically reciprocal to the coupe-stress field and, hence, (ii) concluding that the energy stored in the material through couple-stress action is necessarily identical to the internal energy part that is due to the fibre bending resistance [3].

The fibre-spin vector was employed in [14] as reasonable choice of a dominant spin vector on the grounds that polar material behaviour due to bending resistance of individual fibres can take place in the absence of externally applied couple-stress tractions. Nevertheless, external coupletractions may generally also be present in polar elasticity applications, and, in such cases, no information is there available to guide with precision the choice of a dominant and, therefore, energetically reciprocal rotation/spin field. In this regard, and despite its remarkable success [15], the connection mechanism proposed [14] seems capable to provide only an approximate, though still valuable estimation of the spherical couple-stress.

It the follows that the search for an exact method of spherical couple-stress determination should in fact require direct and proper incorporation of the implied concept of a dominant rotation/spin field into the foundation of the non-linear polar elasticity theory proposed in [1]. This aim then suffices to identify the subject of the present communication, which complements and essentially completes the analysis presented in [1] without dismissing any of the remaining theoretical results detailed or the conclusions drawn there. Instead, it achieves to furnish the theory with additional mathematical means and flexibility that enable it to also determine the spherical couple-stress.

Under these considerations, Section 2 initially outlines the principal postulates and equations that underpin the finite elasticity theory developed in [1], while it also tries to keep unchanged the main notation employed there. Section 3 next introduces the concept of an auxiliary, virtual spin/rotation field whose gradient is energetically reciprocal to the couple-stress field and discusses the physical implications that this innovation brings into the overall, newly proposed theoretical framework.

Section 4 then succeeds to generalise the theory presented in [1] by replacing the general spin field of deformation with the implied auxiliary spin field which, being a virtual such, does not need to be determined. In this manner, a mathematical formulation analogous to that detailed in [1] 
(i) leads again to the sets of stress and couple-stress constitutive equations obtained there, (ii) still predicts the presence of the afore mentioned extra energy term, and (iii) provides a necessary extra equation that enables determination of the spherical couple-stress outside the conventional equilibrium considerations.

Section 4 thus further reveals and discusses the crucial role that the linearized version of the theory plays in the determination of the spherical couple-stress, regardless of whether attention focuses on infinitesimal or finite deformations. Explicit additional details are next provided in Section 5 which, necessarily, restricts attention on relevant, polar elasticity boundary value problems met within the regime of infinitesimal deformations. In this manner, Section 6 is enabled to revisit the simple plate bending problem considered in [15] and, for that specific application, to provide a substantially improved prediction of the spherical couple-stress. Finally, Section 7 summarises the principal conclusions drawn in this communication, elaborates on the ability of the presented, complete form of the theory to determine the spherical couple-stress in relevant finite elasticity applications, and thus reveals and discusses future research directions in this subject.

\section{Notation and preliminary theoretical foundation}

The principal concepts and the notation adopted in [1] will generally still apply in what follows, where all vector and tensor quantities are referred to a rectangular Cartesian coordinate system $\mathrm{O} x_{i}$, indices take the values 1, 2 and 3, and the summation notation of repeated indices also applies. Accordingly, a typical particle that is initially at position $\boldsymbol{X}$, with coordinates $X_{R}$ in the reference configuration, moves to the position $\boldsymbol{x}$, with coordinates $x_{i}$ in the deformed configuration.

The deformation is then described by equations of the form

$x_{i}=x_{i}\left(X_{R}\right)$,

and the deformation gradient tensor, $\boldsymbol{F}$, has components

$F_{i R}=\frac{\partial x_{i}}{\partial X_{R}}=x_{i, R}$,

where, in the usual manner, a comma denotes partial differentiation with respect to the implied coordinate parameter(s). The corresponding right and left Cauchy-Green deformation tensors, $\boldsymbol{C}$ and $\boldsymbol{B}$, have components

$C_{R S}=\frac{\partial x_{i}}{\partial X_{R}} \frac{\partial x_{i}}{\partial X_{S}}=x_{i, R} x_{i, S}, \quad B_{i j}=\frac{\partial x_{i}}{\partial X_{R}} \frac{\partial x_{j}}{\partial X_{R}}=x_{i, R} x_{j, R}$,

respectively.

For a material reinforced by a single family of fibres, a unit vector $\boldsymbol{A}(\boldsymbol{X})$ defines the fibre direction in the reference configuration. The fibres are assumed convected with the material and their direction in the deformed configuration is thus defined by the vector

$b_{i}=F_{i R} A_{R}=\lambda a_{i}, \quad \lambda^{2}=b_{i} b_{i}=A_{R} C_{R S} A_{S}$,

where $\boldsymbol{a}(\boldsymbol{x})$ is a unit vector in the deformed configuration and $\lambda$ denotes the stretch in the fibre direction.

It is noted, for later use, that

$\dot{b_{i}}=\dot{F}_{i R} A_{R}=\frac{\partial \dot{x}_{i}}{\partial X_{R}} A_{R}=\frac{\partial v_{i}}{\partial X_{R}} A_{R}=\frac{\partial v_{i}}{\partial x_{j}} \frac{\partial x_{j}}{\partial X_{R}} A_{R}=\frac{\partial v_{i}}{\partial x_{j}} F_{j R} A_{R}=v_{i, j} b_{j}$,

where a dot denotes differentiation with respect to time and $\boldsymbol{v}$, with components $v_{i}$, is the velocity vector. The corresponding rate of deformation and velocity-spin tensors are

$d_{i j}=\frac{1}{2}\left(v_{i, j}+v_{j, i}\right), \quad \omega_{i j}=\frac{1}{2}\left(v_{i, j}-v_{j, i}\right)$,

Respectively. The latter tensor connects with its own spin vector through the standard relationships 
$\omega_{i j}=\varepsilon_{j i k} \omega_{k}, \quad \omega_{i}=\frac{1}{2} \varepsilon_{i j k} \omega_{k j}=\frac{1}{2} \varepsilon_{i j k} v_{k, j}$,

where $\varepsilon_{i j k}$ are the components of the alternating tensor, $\boldsymbol{\varepsilon}$.

It is further recalled that

$v_{i}=\dot{u}_{i}, \quad u_{i}=x_{i}-X_{i}$,

where $\boldsymbol{u}$ is the displacement vector. The relevant displacement generated spin-field thus is

$\Omega_{i}=\frac{1}{2} \varepsilon_{i j k} \Omega_{k j}=\frac{1}{2} \varepsilon_{i j k} u_{k, j}, \quad \Omega_{k j}=\varepsilon_{i j k} \Omega_{i}$,

where,

$\Omega_{i j}=\frac{1}{2}\left(u_{i, j}-u_{j, i}\right)$

is the standard rotation. Hence,

$\omega_{i j}=\dot{\Omega}_{i j}, \quad \omega_{i}=\dot{\Omega}_{i}$.

The fibres are considered resistant in bending [1]. The strain energy density of the elastic fibre-reinforced material of interest,

$W=W\left(F_{i R}, G_{i R}, A_{R}\right)$,

thus depends not only on the deformation gradients and the fibre direction vector, but also on the gradients

$G_{i R}=\frac{\partial b_{i}}{\partial X_{R}}=b_{i, R}$,

of the deformed fibre tensor. This dependence implies that a couple-stress tensor $\boldsymbol{m}$, with components $m_{\mathrm{ij}}$, is present and, therefore, that the stress tensor $\sigma$, with components $\sigma_{i j}$, is generally non-symmetric. The stress tensor can therefore be decomposed into symmetric and antisymmetric parts in the usual manner, namely

$\sigma_{i j}=\sigma_{(i j)}+\sigma_{[i j]}, \sigma_{(i j)}=\frac{1}{2}\left(\sigma_{i j}+\sigma_{j i}\right), \sigma_{[i j]}=\frac{1}{2}\left(\sigma_{i j}-\sigma_{j i}\right)$.

In the absence of body forces and body couples, the equilibrium equations for the stress and couple-stress are

$\sigma_{j i, j}=0, \quad m_{j i, j}+\varepsilon_{i j k} \sigma_{[j k]}=0$,

respectively. These are accompanied by adequately specified sets of displacement and/or traction and couple-traction boundary conditions acting on the external boundary of the material.

In parts of the boundary that external tractions, $\boldsymbol{T}^{(n)}$, and couple-tractions, $\boldsymbol{L}^{(n)}$, are specified, those boundary conditions relate to (2.15) through the standard Cauchy-type formulas,

$T_{i}^{(n)}=\sigma_{j i} n_{j}, \quad L_{i}^{(n)}=m_{j i} n_{j}$,

where $\boldsymbol{n}$ denotes the outward unit normal of the boundary surface. More generally though, (2.16) also determine the traction and the couple-traction vectors acting on any internal or external surface of the material.

Under the assumption that the components of the stress and the couple-stress tensors are adequately differentiable functions of the co-ordinate parameters, a combination of the pair of equations (2.15) leads to the single equilibrium equation

$\sigma_{(i j), i}+\frac{1}{2} \varepsilon_{k j i} \bar{m}_{\ell k, \ell i}=0$,

where

$\bar{m}_{\ell k}=m_{\ell k}-\frac{1}{3} m_{r r} \delta_{\ell k}$ 
is the deviatoric part of the couple-stress tensor, and the appearing Kronecker's delta stands for the components of the unit matrix.

It is thus seen that the spherical couple-stress, $m_{r r}$, does not influence the equilibrium and, therefore, cannot be determined through the outlined standard equilibrium considerations. The noted indeterminacy of $m_{r r}$ is a long-known weakness of the conventional Cosserat couple-stress theory (e.g., [4-13]) and, as such, it is also present in the finite elasticity modelling effort detailed earlier in [1] for composite materials reinforced by fibres resistant in bending. As is shown next though, the theory developed in [1] is susceptible to appropriate generalisation and, hence, completion that enables determination of $m_{r r}$.

\section{Virtual and actual rotation and spin fields}

Conventional couple-stress theory [2] considers that external couple-tractions that may act on the boundary surface of a polar elastic solid produce work, and thus store energy in the material, through their interaction with the gradients of the deformation generated spin-field (2.9a). This consideration might be felt trivially valid in the case of material isotropy but can be misleading in the presence of material anisotropy that is due to fibre reinforcement. This is because the relevant fibre rotation/spin field, which be an alternative or additional source of couple-stress, can differ to its deformation counterpart (2.9a). It is noted in this regard that, in the absence of externally applied, boundary couple-tractions, polar material behaviour in a fibre-reinforced material can still be triggered, solely, through fibre deformation and spin.

It thus becomes understood that, if present, boundary couple-tractions store energy in the deforming material through their interaction with some auxiliary spin/rotation field,

$\Phi_{i}=\frac{1}{2} \varepsilon_{i j k} \Phi_{k j}, \quad \Phi_{k j}=\varepsilon_{i j k} \Phi_{i}$,

which may generally represent some unknown combination of its fibre and general deformation counterparts. The rates

$\psi_{i j}=\dot{\Phi}_{i j}, \quad \psi_{i}=\dot{\Phi}_{i}$,

of the implied rotation tensor and spin vector are then naturally related as follows:

$\psi_{i j}=\varepsilon_{j i k} \psi_{k}, \quad \psi_{i}=\frac{1}{2} \varepsilon_{i j k} \psi_{k j}$.

For reasons that will become clear in what follows, $\psi_{i}$ is now required to satisfy the condition

$\left(m_{j i} \psi_{i}\right)_{, j}=\left(m_{j i} \omega_{i}\right)_{, j}, \quad\left(\psi_{i} \neq \omega_{i}\right)$.

The special case in which $\psi_{i}=\omega_{i}$ is naturally regarded as an uninteresting, trivial solution of (3.4) that makes the present analysis identical to its counterpart detailed in [1].

It is emphasised that, on its own, the single condition (3.4) does not suffice for unique determination of $\psi_{i}$. Indeed, (3.4) may be used for determination of one component of $\psi_{i}$, but only after the other two components of this vector are specified arbitrarily. Hence, unless a couple of additional conditions somehow emerge to accompany (3.4), there exists a double-infinite number of vectors $\psi_{i}$ that satisfy (3.4). In this context, the auxiliary spin vector (3.3b) represents a virtual spin field.

Within the standard framework that underpins the principle of virtual work (e.g., $[16,17])$, it is now recalled that virtual velocities and, hence, their velocity-spin fields of the type implied in (3.4), are replaceable by their virtual displacements and, hence, displacement-spin counterparts (3.1a) 
and (2.9a), respectively. Within that framework, condition (3.4) thus is replaceable and, therefore, equivalent with the condition

$\left(m_{j i} \Phi_{i}\right)_{, j}=\left(m_{j i} \Omega_{i}\right)_{, j}, \quad\left(\Phi_{i} \neq \Omega_{i}\right)$,

which is valid for all relevant virtual displacements, including their real displacements, and regardless of whether the deformation is large or small (e.g., [16]).

The physical meaning of (3.4) and (3.5) can become better understood by integrating the latter condition over an arbitrary volume, $V$, of the solid material of interest and making afterwards use of the divergence theorem. That process yields

$\int_{V}\left[m_{j i}\left(\Phi_{i}-\Omega_{i}\right)\right]_{, j} d V=\int_{S} m_{j i}\left(\Phi_{i}-\Omega_{i}\right) n_{j} d S=0$,

which, with use of (2.16b) leads to

$\int_{S} L_{i}^{(n)} \Phi_{i} d S=\int_{S} L_{i}^{(n)} \Omega_{i} d S$

where $S$ represents the bounding surface of $V$.

Validity of (3.4) or, equivalently, (3.5) thus implies that the total work done by the set of boundary couple-tractions, $L_{i}^{(n)}$, acting on the virtual spin vector, $\Phi_{i}$, equals the total work done by the same set of couple-tractions acting on the actual displacement-spin vector, $\Omega_{i}$. In this regard, it is also noted that either side of (3.5) has dimensions of energy per unit volume. While the right-hand side of (3.5) represents energy per unit volume due to interaction of the couple-stress tensor with the actual, displacement generated spin vector, the left-hand side is an equal amount of energy density that is due to interaction of the couple-stress with a virtual displacement-spin.

\section{Refined finite elasticity of fibre-reinforced materials when fibres resist bending}

A straightforward generalisation/refinement of the modelling process that led to the constitutive equations derived in [1] can now begin by (i) confining again attention to quasi-static deformations only, (ii) considering that (2.12) and (2.16) still hold, but (iii) modifying the energy balance equation as follows:

$$
\frac{D}{D t} \int_{V} W d V=\int_{S}\left(T_{i}^{(n)} v_{i}+L_{i}^{(n)} \psi_{i}\right) d S,
$$

where $\psi_{i}$ replaces the velocity-spin vector $(2.7 \mathrm{~b})$ employed in [1].

Application of Reynolds transport theorem and the divergence theorem next yields

$$
\frac{\rho}{\rho_{0}} \dot{W}=\sigma_{j i} v_{i, j}+\left(m_{j i} \psi_{i}\right)_{, j} \text {, }
$$

where $\rho$ and $\rho_{0}$ denote material density in the deformed and the reference configuration, respectively, and the equilibrium equation (2.15a) is also accounted for. Hence, use of (2.6), (2.7), (2.14), (2.15b) and (3.3) leads to

$$
\begin{aligned}
\frac{\rho}{\rho_{0}} \dot{W} & =\sigma_{(j i)} d_{i j}+\sigma_{[j i]} \omega_{i j}-\varepsilon_{i j k} \sigma_{[j k]} \psi_{i}+m_{j i} \psi_{i, j} \\
& =\sigma_{(j i)} d_{i j}+\sigma_{[j i]} \varepsilon_{k j i}\left(\omega_{k}-\psi_{k}\right)+m_{j i} \psi_{i, j} \\
& =\sigma_{(j i)} d_{i j}+m_{j i, j}\left(\psi_{i}-\omega_{i}\right)+m_{j i} \psi_{i, j} .
\end{aligned}
$$

On the other hand, the form (2.12) of the strain energy density still enables one to obtain 
$\dot{W}=\left(F_{i R} \frac{\partial W}{\partial F_{j R}}+G_{i R} \frac{\partial W}{\partial G_{j R}}\right)\left(d_{i j}+\omega_{i j}\right)+F_{j R} \frac{\partial W}{\partial G_{i R}} b_{k} v_{i, j k}$.

This equation is identical to Eq. (5.15) obtained in [1], and its derivation process is not repeated here. However, its comparison with (4.3) now leads to the relationship

$$
\begin{aligned}
\left\{\sigma_{(j i)}-\frac{\rho}{\rho_{0}}\left(F_{i R} \frac{\partial W}{\partial F_{j R}}\right.\right. & \left.\left.+G_{i R} \frac{\partial W}{\partial G_{j R}}\right)\right\} d_{i j}-\frac{\rho}{\rho_{0}}\left(F_{i R} \frac{\partial W}{\partial F_{j R}}+G_{i R} \frac{\partial W}{\partial G_{j R}}\right) \omega_{i j} \\
& +\left[m_{j i, j}\left(\psi_{i}-\omega_{i}\right)\right]_{, j}+m_{j i} \omega_{i, j}-\frac{\rho}{\rho_{0}} F_{j R} \frac{\partial W}{\partial G_{i R}} b_{k} v_{i, j k}=0,
\end{aligned}
$$

which is slightly different to its counterpart derived in [1].

Since $\psi_{i}$ is a virtual, essentially arbitrary spin vector, condition (3.4) emerges at this point as a necessary requirement that enables (4.5) to hold in a meaningful mathematical sense. Nevertheless, when combined with (3.4), equation (4.5) becomes identical with its counterpart noted as (5.18) in [1], namely with the equation that the whole analysis detailed in [1] is based upon. In this context, the whole set of results presented in [1] becomes naturally obtainable with use of the present analysis.

\subsection{Principal theoretical results that follow the combination of (3.4) and (4.5)}

It then becomes necessary, mainly for later use, to briefly quote some of the principal results obtained in [1] without repeating relevant derivation details. Accordingly, the combination of (4.5) and (3.4) leads again to the constitutive equations [1]

$$
\begin{aligned}
& \sigma_{(j i)}=\frac{\rho}{\rho_{0}}\left(F_{i R} \frac{\partial W}{\partial F_{j R}}+G_{i R} \frac{\partial W}{\partial G_{j R}}\right), \\
& \bar{m}_{j r}=\frac{2}{3} \varepsilon_{r k i} \frac{\rho}{\rho_{0}} \frac{\partial W}{\partial G_{i R}}\left(F_{j R} b_{k}+F_{k R} b_{j}\right), \quad \bar{m}_{k k}=0,
\end{aligned}
$$

where $W$ is of the form (2.12) and the appearing symmetric part of stress and deviatoric couple-stress are defined in (2.14b) and (2.18), respectively. It is recalled that (4.6b) leaves the spherical couplestress indetermined, though, in line with other relevant earlier developments (e.g., [4-13]), this result was not considered as surprising in [1].

It is further recalled [1] that the required invariance of the strain energy density in rigid rotation of the co-ordinate system is satisfied if

$W=W(\boldsymbol{C}, \boldsymbol{\Lambda}, \boldsymbol{A})$,

where the appearing Cauchy-Green deformation tensor is defined in (2.3a) and the tensor $\boldsymbol{A}=\boldsymbol{F}^{\boldsymbol{T}} \boldsymbol{G}$ has components

$$
\Lambda_{R S}=\frac{\partial x_{i}}{\partial X_{R}} \frac{\partial b_{i}}{\partial X_{S}}=F_{i R} G_{i S} \text {. }
$$

With $W$ being re-casted in the form (4.7), the constitutive equations (4.6) are also reformed and become

$$
\begin{aligned}
& \sigma_{(j i)}=\frac{\rho}{\rho_{0}}\left\{F_{i R} F_{i S}\left(\frac{\partial W}{\partial C_{R S}}+\frac{\partial W}{\partial C_{S R}}\right)+\left(G_{i R} F_{j S}+G_{j R} F_{i S}\right) \frac{\partial W}{\partial \Lambda_{S R}}\right\}, \\
& \bar{m}_{j i}=\frac{2}{3} \varepsilon_{i k m} \frac{\rho}{\rho_{0}} \frac{\partial W}{\partial \Lambda_{P R}} F_{m P}\left(F_{j R} b_{k}+F_{k R} b_{j}\right), \quad \bar{m}_{k k}=0 .
\end{aligned}
$$

Connection of these constitutive equations with the equations of equilibrium (2.17) thus provides all the principal information needed for determination of the deformation, as well as of the stress and the deviatoric part of the couple-stress. Nevertheless, the extra piece of information that 
(3.4) made available in the present refined formulation can still be used for determination of the spherical couple-stress outside the standard equilibrium conventions.

Towards this end, it is further recalled [1] that, since the strain energy density (4.7) is an isotropic invariant of the tensors $\boldsymbol{C}, \boldsymbol{\Lambda}$ and the vector $\boldsymbol{A}$, it is expressible in the form

$W=W\left(I_{1}, I_{2}, \ldots, I_{33}\right)$,

as a function of thirty-three conventional deformation invariants, whose full list is provided in the Appendix of [1]. It is noted in passing that the number of elements of that list is reduced to eleven in the case of a restricted version of the theory (see Section 6 of [1]) but that reduction does not affect the principal argument that underpins the below proposed method of determination of the spherical couple-stress.

That argument follows the standard transformation that (4.9) attain by virtue of (4.10), namely [1]

$$
\begin{aligned}
& \sigma_{(j i)}=\frac{\rho}{\rho_{0}} \sum_{\ell=1}^{33} \frac{\partial W}{\partial I_{\ell}}\left\{F_{i R} F_{i S}\left(\frac{\partial I_{\ell}}{\partial C_{R S}}+\frac{\partial I_{\ell}}{\partial C_{S R}}\right)+\left(G_{i R} F_{j S}+G_{j R} F_{i S}\right) \frac{\partial I_{\ell}}{\partial \Lambda_{S R}}\right\}, \\
& \bar{m}_{j i}=\frac{2}{3} \varepsilon_{i k m} \frac{\rho}{\rho_{0}} \sum_{\ell=1}^{33} \frac{\partial W}{\partial I_{\ell}} \frac{\partial I_{\ell}}{\partial \Lambda_{P R}} F_{m P}\left(F_{j R} b_{k}+F_{k R} b_{j}\right), \quad \bar{m}_{k k}=0,
\end{aligned}
$$

and is underpinned by the role that the invariant

$I_{20}=\boldsymbol{A} \boldsymbol{\Lambda} \boldsymbol{A}$

plays in the outlined formulation. It is accordingly already observed in [1] that, although $I_{20}$ is associated with deformation features stemming, purely, from fibre bending resistance, this invariant fails to relate its strain energy contribution with the couple-stress constitutive equation (4.11b).

\subsection{The role of the invariant $I_{20}$ and its strain energy contribution}

Indeed, by combining the following intermediate result:

$\frac{\partial I_{20}}{\partial \Lambda_{P R}}=\frac{\partial\left(A_{M} \Lambda_{M N} A_{N}\right)}{\partial \Lambda_{P R}}=\delta_{M P} \delta_{N R} A_{M} A_{N}=A_{P} A_{R}$,

with the properties of the alternating tensor, someone obtains

$\varepsilon_{i k m} \frac{\partial W}{\partial I_{20}} \frac{\partial I_{20}}{\partial \Lambda_{P R}} F_{m P}\left(F_{j R} b_{k}+F_{k R} b_{j}\right)=\frac{\partial W}{\partial I_{20}} \varepsilon_{i k m} F_{m P} A_{P}\left(F_{j R} b_{k}+F_{k R} b_{j}\right) A_{R}=2 \frac{\partial W}{\partial I_{20}} \varepsilon_{i k m} b_{m} b_{k} b_{j}=0$.

It is thus seen that (i) no part of the strain energy that may depend solely on $I_{20}$ can contribute into the deviatoric coupe-stress formation and, subsequently, (ii) no part of the strain energy density that depends purely on $I_{20}$ is created through action of the deviatoric couple-stress (4.11b). However, $I_{20}$ still stems solely from fibre deformation resistance. It necessarily follows that such a part, $W^{m}\left(I_{20}\right)$ say, of stored energy can only be related to action of the spherical couple-stress.

A counter argument might emerge at this point, claiming that the $I_{20}$-contribution noted in the strain energy density is relevant to the symmetric part of the stress tensor only, through the involvement of $I_{20}$ in the second term appearing within the curled bracket of (4.11a). However, such a counter argument is dismissed by initially observing that that term is of the second order in the strains (see also Section 9 of [1]). Its influence is, therefore, negligible within the small deformation regime, where only the first term appearing in the same bracket is linear and, hence, offers contribution.

Within the infinitesimal deformation regime, where the strain energy function is necessarily quadratic in its arguments, the stress constitutive equation (4.11a) thus becomes completely 
uncoupled from its couple-stress counterpart (4.11b) and, as a result, the contribution of $I_{20}$ influences only the couple-stress constitutive equation (see also [3, 15] as well as Sections 5 and 6 below). Potential validity of the afore mentioned counter argument is thus dismissed because, within the infinitesimal deformation regime, the strain energy function contains a term of the form $W^{m}\left(I_{20}\right)$ that is necessarily quadratic in $I_{20}$.

It is recalled in this context that any form of a strain energy density employed in finite elasticity applications is required to be consistent with its own approximate form that serves the purposes of the linear, infinitesimal deformation version of the theory. The noted connection of $I_{20}$ with the energy contribution of the spherical couple-stress is therefore anticipated generally present not only within the infinitesimal, but also within the large deformation regime where, as is already seen, its influence on the constitutive equations is of a higher-order in the strains.

The implied quadratic form of $W^{m}\left(I_{20}\right)$, which otherwise emerges inexplicably in the small deformation regime, is accordingly regarded as a leading order approximation of the influence that the spherical couple-stress exerts on any form of a corresponding strain energy density employed in finite elasticity applications. It follows that a term of the form $W^{m}\left(I_{20}\right)$ is anticipated present in the form of any relevant, admissible strain energy density or in its equivalent polynomial expansion in terms of the invariants. Alternatively, the leading-order (small strain) approximation of the $W^{m}$-term, which must be present in any admissible strain energy density, must necessarily be quadratic in $I_{20}$.

\subsection{Determination of the spherical part of the couple-stress tensor}

It is now recalled that each side of (3.5) represents some unknown amount of energy per unit volume formed through the divergence of the interaction the couple-stress tensor with the noted spin vector. By virtue of (3.4) and (4.5), the implied amount of energy does not influence the constitutive equations and, like $W^{m}$, does not influence the state of equilibrium. It follows that, necessarily,

$\left(m_{j i} \Phi_{i}\right)_{, j}=\left(m_{j i} \Omega_{i}\right)_{, j}=W^{m}\left(I_{20}\right)$.

It is then observed that, unless the essentially unknown, virtual spin field $\Phi_{i}$ is specified in some artificial technical manner (e.g., [15]), the left-hand side of (4.15) cannot possibly employed to offer any kind of a useful relevant information. However, a combination of the remaining of (4.15) with (2.18) yields

$\Omega_{j} m_{k k, j}=3 W^{m}\left(I_{20}\right)-3\left(\bar{m}_{j i} \Omega_{i}\right)_{, j}$,

which is a first-order partial differential equation (PDE), for the unknown spherical part of the couple-stress, $m_{k k}$. Solution of this PDE, subject to an appropriate subset of couple-traction boundary conditions, will thus lead to determination of $m_{k k}$ and, hence, to complete determination of the couple-stress tensor.

Precise determination of the virtual spin vector $\Phi_{i}$ thus becomes unnecessary. Nevertheless, some auxiliary, technical choice of $\Phi_{i}$ could be found advantageous in special cases or applications (e.g., $[14,15])$ where, however, relevant theoretical predictions are now regarded as approximations of their counterparts based on the present development.

In conclusion, the outlined refined formulation of the theory presented in [1] enables determination of the spherical couple-stress by essentially leaving indeterminate or unknown the spin vector that the externally applied boundary couple-tractions choose to act upon. This is though considered as a minor indeterminacy issue, in the sense that it does not anymore prevent complete determination of the deformation as well as the couple-stress and the non-symmetric stress fields. 


\section{Small deformations}

In the light of the observations made and the conclusions drawn in the last couple of paragraphs of Section 4.2, attention is now necessarily directed on the linearized version of the theory. It is accordingly recalled [3] that within the small deformation regime, where strains and curvature-strains are considered of the same, infinitesimal order of magnitude, the constitutive equations (4.9) attain the simplified form

$$
\sigma_{(i j)}=\frac{\partial W}{\partial e_{i j}}, \quad \bar{m}_{\ell r}=\frac{2}{3} \varepsilon_{r s i}\left(\frac{\partial W}{\partial \kappa_{i \ell}} a_{s}+\frac{\partial W}{\partial \kappa_{i s}} a_{\ell}\right), \quad \bar{m}_{k k}=0
$$

where the appearing components of the infinitesimal strain and curvature-strain tensors, $\boldsymbol{e}$ and $\boldsymbol{\kappa}$, are respectively

$$
e_{i j}=\frac{1}{2}\left(u_{i, j}+u_{j, i}\right), \quad \kappa_{i j}=\left(u_{i, k} a_{k}\right)_{, j},
$$

and, since the reference and the deformed configurations are considered identical, the standard simplified notation $\boldsymbol{a} \equiv \boldsymbol{A}$ is also employed.

Moreover, the strain energy function that approximates in the small deformation regime any admissible form of a strain energy density is decomposed into two independent parts as follows:

$W(\mathbf{e}, \boldsymbol{\kappa}, \mathbf{a})=W^{e}(\mathbf{e}, \mathbf{a})+W^{\kappa}\left(\boldsymbol{\kappa}_{s}, \boldsymbol{\kappa}_{a}, \mathbf{a}\right)$.

Here, $\boldsymbol{\kappa}_{s}$ and $\boldsymbol{\kappa}_{\boldsymbol{a}}$, with components

$$
\begin{aligned}
& \kappa_{(i j)}=\frac{1}{2}\left(\kappa_{i j}+\kappa_{j i}\right)=\frac{1}{2}\left[\left(u_{i, k} a_{k}\right)_{, j}+\left(u_{j, k} a_{k}\right)_{, i}\right] \\
& \kappa_{[i j]}=\frac{1}{2}\left(\kappa_{i j}-\kappa_{j i}\right)=\frac{1}{2}\left[\left(u_{i, k} a_{k}\right)_{, j}-\left(u_{j, k} a_{k}\right)_{, i}\right],
\end{aligned}
$$

stand for the symmetric and the anti-symmetric parts of $\boldsymbol{\kappa}$, respectively, while $W^{e}$ and $W^{\kappa}$ are both even in $\boldsymbol{a}$ and necessarily strictly quadratic in their arguments.

It follows $[1,3]$ that $W^{e}$ is necessarily identical with its transversely isotropic counterpart met in non-polar linear elasticity and, by virtue of (5.1a), so it is the constitutive equation that provides the symmetric part of the stress tensor.

On the other hand, the most general relevant form of $W^{\kappa}$ is as follows:

$$
\begin{aligned}
W^{\kappa}=\beta_{1}\left(\kappa_{n n}\right)^{2} & +\beta_{2} \kappa_{n n} a_{k} \kappa_{(k m)} a_{m}+\beta_{3} \kappa_{(k m)} \kappa_{(m k)}+\beta_{4} a_{k} \kappa_{(k m)} \kappa_{(m n)} a_{n} \\
& +\beta_{5} \kappa_{[k m]} \kappa_{[m k]}+\beta_{6} a_{k} \kappa_{[k m]} \kappa_{[m n]} a_{n}+\beta_{7} a_{k} \kappa_{(k m)} \kappa_{[m n]} a_{n}+\hat{\beta}_{3}\left(a_{k} \kappa_{(k m)} a_{m}\right)^{2},
\end{aligned}
$$

and, for this to be positive definite, the appearing coefficients, $\beta_{1}-\beta_{7}$ and $\hat{\beta}_{3}$, must satisfy the following inequalities [3]:

$\beta_{1} \geq 0, \quad \beta_{3} \geq 0, \quad \beta_{5} \leq 0, \quad \beta_{2}+\hat{\beta}_{3} \geq 0, \quad \beta_{1}+\beta_{2}+\beta_{3}+\beta_{4}+\hat{\beta}_{3} \geq \frac{\left(\beta_{1}+\beta_{2} / 2\right)^{2}}{\beta_{1}+\beta_{3}}$.

By virtue of (5.1a), the linear couple-stress constitutive equation then obtains the more specific form

$$
\begin{aligned}
\bar{m}_{\ell r}= & \frac{2}{3} \varepsilon_{r \ell s} a_{s}\left(2 \beta_{1} \kappa_{n n}+\beta_{2} \kappa_{k m} a_{k} a_{m}\right)+\frac{2}{3} \varepsilon_{r i s} a_{s}\left(2 \beta_{3} \kappa_{(i \ell)}+\beta_{4} \kappa_{(i n)} a_{n} a_{\ell}\right)- \\
& \frac{1}{3} \varepsilon_{r i s}\left\{4 \beta_{5}\left(a_{s} \kappa_{[i \ell]}+a_{\ell} \kappa_{[i s]}\right)-2 \beta_{6} a_{n} a_{\ell}\left(a_{i} \kappa_{[s n]}-2 a_{s} \kappa_{[i n]}\right)+\beta_{7} a_{n} a_{\ell}\left(a_{i} \kappa_{n s}-2 a_{s} \kappa_{i n}\right)\right\},
\end{aligned}
$$


which reveals that the energy term that involves $\hat{\beta}_{3}$ in (5.5) is not related with the action of the deviatoric couple-stress. This thus is the afore mentioned extra energy term,

$W^{m}=\hat{\beta}_{3}\left(a_{k} \kappa_{(k n)} a_{n}\right)^{2}$,

that represents energy stored in the material through sole action of the spherical couple-stress. $W^{m}$ must then be also positive definite, and this requirement adds on (5.6) the inequality

$\hat{\beta}_{3}>0$.

For any relevant, well-posed boundary value problem, connection of the constitutive equations stemming form (5.1a) and (5.7) with the equations of equilibrium (2.17) will generally provide all the information needed for determination of the deformation, as well as the symmetric part of stress and the deviatoric couple-stress. That information enables subsequent determination of the extra energy term (5.8) and, hence, of a corresponding, specific form of the PDE (4.16). Solution of that PDE, subject to the relevant subset of couple-stress boundary conditions, will finally determine the spherical couple-stress.

\subsection{The restricted, splay-mode version of the linear theory}

A simplified version of the outlined linear theory refers to boundary value problems anticipating that the couple-stress part, $W^{\kappa}$, of the strain function is predominantly influenced by energy contributions due to splay-type of fibre-deformation. This version of the theory is based on the simplifying consideration [18]

$W^{\kappa}\left(\boldsymbol{\kappa}_{s}, \boldsymbol{\kappa}_{a}, \boldsymbol{a}\right)=W^{\kappa}\left(\boldsymbol{\kappa}_{a}, \boldsymbol{\kappa}_{s}, \boldsymbol{a}\right)=W^{\kappa}(\boldsymbol{\kappa}, \boldsymbol{a})$,

and thus enables reduction of (5.5) into the following form:

$$
\begin{aligned}
W^{\kappa} & =\beta_{1}\left(\kappa_{n n}\right)^{2}+\beta_{2} \kappa_{n n} a_{k} \kappa_{k m} a_{m}+\hat{\beta}_{3}\left(a_{k} \kappa_{k m} a_{m}\right)^{2} \\
& =\beta_{1}\left(\kappa_{(n n)}\right)^{2}+\beta_{2} \kappa_{(n n)} a_{k} \kappa_{(k m)} a_{m}+\hat{\beta}_{3}\left(a_{k} \kappa_{(k m)} a_{m}\right)^{2},
\end{aligned}
$$

which makes use of three elastic moduli only.

Subsequently, the corresponding couple-stress constitutive equation is also simplified and becomes [18]

$\bar{m}_{\ell r}=\frac{2}{3} \varepsilon_{r \ell s} a_{s}\left(2 \beta_{1} \kappa_{n n}+\beta_{2} \kappa_{k m} a_{k} a_{m}\right)=\frac{2}{3} \varepsilon_{r \ell s} a_{s}\left(2 \beta_{1} \kappa_{(n n)}+\beta_{2} \kappa_{(k m)} a_{k} a_{m}\right)$,

thus making use of only two of those elastic moduli. Clearly, this constitutive equation considers that, when fibre-splay deformation dominates, the first term in the right-hand side of (5.7) provides enough information for accurate determination of the deviatoric couple-stress.

Moreover, like (5.7), (5.12) still suggests that the deviatoric couple-stress is essentially irrelevant to the energy term involving $\hat{\beta}_{3}$ and thus leads again to (5.8). The spherical part of the couple-stress can then still be determined in the manner described in the previous section.

\subsection{The restricted, bending-mode version of the linear theory}

This is the earliest of the two simplified versions of the unrestricted linear theory and has already been employed in a series of specific boundary value problem applications [18-22]. It was initially obtained in [3] through direct linearisation of the relevant, restricted, non-linear theory presented in Section 5 of [1]. That version of the theory makes use of a plausible consideration that excludes from the strain energy density/function energy contributions associated with fibre-splay and fibre-twist deformation effects and thus promotes the physically reasonable claim that, in fibre composite solids, fibre bending and curvature are generally the major relevant factors.

The implied linearisation process [3] thus led to the simplest possible form of (5.5), namely 
$W^{\kappa}=\frac{3}{8} d^{f} K_{j} K_{j}+\bar{\gamma}\left(a_{j} K_{j}\right)^{2}, \quad K_{i}=u_{i, k j} a_{k} a_{j}$.

This makes use of two elastic moduli only and it is positive definite only if the values of these moduli are both positive, namely

$d^{f}>0, \quad \bar{\gamma}>0$.

equation,

$\bar{m}_{\ell r}=d^{f} \varepsilon_{r s i} K_{i} a_{\ell} a_{s}$,

thus leading to the conclusion that, in this case, it is

$W^{m}=\bar{\gamma}\left(a_{i} K_{i}\right)^{2}=\bar{\gamma}\left(u_{i, k j} a_{i} a_{k} a_{j}\right)^{2}$.

After the appearing displacement vector is determined in a particular application, and its secondorder partial derivatives are appropriately inserted into (5.16), the resulting expression of $W^{m}$ can be inserted into (4.16). As is already mentioned, solution of the thus obtained PDE will determine the spherical couple-stress.

\section{Application: Pure bending of a rectangular plate reinforced by a family of straight fibres}

This boundary value problem is among the simplest problems that the outlined analysis can be applied to, and thus is already employed for relevant pilot studies in previous relevant investigations $[15,23]$. As is already mentioned in the Introduction, Reference [15] employed this problem in a successful initial attempt to obtain an estimate of the spherical-couple stress, through use of a technique that combines the relevant form attained by the strain energy function with a suitable generalisation of the conventional couple-stress theory [14]. Despite its remarkable success [15], and for reasons detailed already in Section 4.3 above, the afore-mentioned connection mechanism [14] seems now capable to provide only an approximate estimation of the spherical couple-stress.

However, it is precisely the valuable experience gained through the successful application of that mechanism [14] that led to the new developments detailed in this communication. Since, as is already mentioned, this is based on a properly constructed generalisation of the non-linear theory presented in [1] (Section 3 above), the results of the spherical couple-stress determination detailed in the present Section are naturally more accurate to those obtained in [15].

Details of the history of the plate bending problem of present interest as well as of its solution are provided in [15]. A brief recollection of the latter may begin with a reminder of the plate nomenclature provided in Figure 1, as well as of the fact that the embedded straight fibres are aligned with the $x_{1}$-direction of the depicted Cartesian co-ordinate system, so that

$\boldsymbol{a}=(1,0,0)^{T}$.

The plate is subjected to pure bending through action of a normal stress and a shear couplestress distribution

$\sigma_{11}=\hat{\sigma}_{1} x_{2}, \quad \bar{m}_{13}=-\hat{m}_{3}$,

respectively. As is shown in Figures 2 and 3, these boundary tractions are applied externally on the edges $x_{1}= \pm L_{1}$, with $\hat{\sigma}_{1}$ and $\hat{m}_{3}$ been regarded as known positive constants. No other stress or shear couple-stress distributions are applied externally on any of the six boundary planes of the plate. However, normal couple-stress distributions may still be applicable externally on the plate boundaries, but their proper consideration is not possible before the spherical couple-stress is determined.

The exact solution of this polar linear elasticity boundary value problem requires from (6.2) to be the only non-zero stress and deviatoric shear couple-stress components acting not only on the 
edges $x_{1}= \pm L_{1}$, but also throughout the whole body of the plate [15]. The corresponding displacement field is then given as follows:

$u_{1}=\hat{\sigma}_{1} S_{11} x_{1} x_{2}, \quad u_{2}=\frac{1}{2} \hat{\sigma}_{1}\left[S_{12}\left(x_{2}^{2}-x_{3}^{2}\right)-S_{11} x_{1}^{2}\right], \quad u_{3}=\hat{\sigma}_{1} S_{12} x_{3} x_{2}$,

where $S_{11}$ and $S_{12}$ represent the elastic compliances met in the corresponding non-polar elasticity problem. Those compliances are obtained in the usual manner, by inverting the corresponding matrix of elastic stiffnesses.

It is worth mentioning that the displacement field (6.3) is seemingly independent of the magnitude, $\hat{m}_{3}$, of the externally applied shear couple-stress. Nevertheless, equations (6.10) and (6.16) below will make evident that $\hat{m}_{3}$ and $\hat{\sigma}_{1}$ are not independent constants. Knowledge of the plate displacement field enables determination of the corresponding strain, curvature-strain, and displacement-spin fields and, through use of the couple-stress constitutive equations, can finally lead to the determination of the spherical couple-stress.

The relevant process requires direct use of the displacement-spin vector stemming from (2.9) and (2.10), namely

$\boldsymbol{\Omega}=\left(\omega_{32}, \omega_{13}, \omega_{21}\right)^{T}=\left(\hat{\sigma}_{1} S_{12} x_{3}, 0,-\hat{\sigma}_{1} S_{11} x_{1}\right)^{T}$,

and is next demonstrated for two of the afore mentioned three linear versions of the theory. The remaining version of the theory, namely the version that is predominantly interested on the fibresplay deformation mode, does not need to be considered because, as is detailed in [15], fibre-splay deformations are totally absent in this pure bending application.

\subsection{The fibre-bending deformation mode/version of the theory}

In this relatively simpler version of the theory, a combination of (5.13b) with (6.1) and (6.3) yields $K_{1}=K_{3}=0, \quad K_{2}=u_{2,11}=-\hat{\sigma}_{1} S_{11}$.

Hence, (5.13a) reveals that the curvature part of the strain energy function is

$W^{\kappa}=\frac{3}{8} d^{f} K_{2}^{2}=\frac{3}{8} d^{f}\left(\hat{\sigma}_{1} S_{11}\right)^{2}$,

while (5.16) returns

$W^{m}=0$.

On the other hand, a combination of (6.5) and (6.1) with (5.15) reveals that the only nonzero component of the deviatoric couple-stress tensor is

$\bar{m}_{13}=d^{f} K_{2}=-d^{f} \hat{\sigma}_{1} S_{11}$.

Since every normal component of the deviatoric couple-stress thus is zero $\left(\bar{m}_{11}=\bar{m}_{22}=\bar{m}_{33}=0\right)$, (2.18) yields

$m_{11}=m_{22}=m_{33}=\frac{1}{3} m_{r r}$.

Moreover, a comparison of (6.8) with (6.2b) makes it understood that the positive constants, $\hat{\sigma}_{1}$ and $\hat{m}_{3}$, that regulate the implied pure plate bending are necessarily connected through the relationship

$\hat{m}_{3}=\hat{\sigma}_{1} d^{f} S_{11}$,

and, hence, cannot be chosen independently in some arbitrary manner.

The spherical couple-stress, $m_{k k}$, can now be determined by solving the PDE (4.16) which, with use of (6.4), (6.7), (6.8) and (6.10), obtains the specific form 
$\frac{S_{12}}{S_{11}} x_{3} m_{r r, 1}-x_{1} m_{r r, 3}=-\frac{15}{8} \hat{m}_{3}$.

There is remarkable similarity of this differential equation with its counterpart noted as Eq. (4.12) in [15] which, however, has a zero right-hand side and is, therefore, a homogeneous PDE. The observed dependence of the non-zero right-hand side of (6.11) from the externally applied couple-stress distribution thus is recognised as a product of additional sensitivity and accuracy that distinguishes the present mathematical model from its counterpart proposed in [14].

As is detailed in the Appendix, the method of characteristic lines yields the general solution of (6.11) as follows:

$$
\begin{aligned}
& \frac{x_{1}^{2}}{S_{12}}+\frac{x_{3}^{2}}{S_{11}}=\lambda^{2}\left(x_{2}\right), \\
& m_{k k}\left(x_{1}, x_{2}, x_{3}\left(x_{1}\right)\right)=\mp \frac{15 \hat{m}_{3}}{8} \sqrt{\frac{S_{11}}{S_{12}}} \sin ^{-1}\left(\frac{x_{1}}{\sqrt{S_{12}} \lambda\left(x_{2}\right)}\right)+c\left(x_{2}\right),
\end{aligned}
$$

where $\lambda\left(x_{2}\right)$ and $c\left(x_{2}\right)$ are arbitrary functions of integration. It is immediately noted that, while the characteristic lines (6.12a) are still elliptical curves, $(6.12 \mathrm{~b})$ is a substantially more specific and, therefore, more accurate representation of the spherical couple-stress than its counterpart noted as (4.13) in [15].

Connection of (6.12) with (6.9) thus implies that the considered, pure bending plate deformation pattern is sustainable only if some additional set of boundary conditions is specified on the plate outer planes. That set is evidently relevant to the boundary values that each one of the normal couple-stresses attains on the relevant pair of boundary planes and, as its possible specification is not unique, will not be pursued much further.

It should be sufficient though to mention that if, for instance, it is assumed that

$$
\left.m_{11}\right|_{x_{1}= \pm L_{1}}=0 \text {, }
$$

then a combination of (6.9) and (6.12) makes it evident that the functions $\lambda\left(x_{2}\right)$ and $c\left(x_{2}\right)$ must be related as follows:

$\left[\lambda\left(x_{2}\right)\right]^{-1}=\frac{\sqrt{S_{12}}}{L_{1}} \sin \left[\frac{8}{15 \hat{m}_{3}} \sqrt{\frac{S_{12}}{S_{11}}} c\left(x_{2}\right)\right]$,

and, hence, that only one of these functions can be chosen arbitrarily. In this manner, a boundary condition of the type (6.13) essentially restricts the range of values that the other two normal couplestresses can attain on their respective boundary planes.

\subsection{Unrestricted theory}

In the case of the unrestricted theory, a combination of (6.1) with (5.2b) yields

$\kappa_{i j}=u_{i, j 1}$.

Connection of this relationship with (6.3) forces (5.7) to return only a single non-zero component of the deviatoric couple-stress, namely

$\bar{m}_{13}=\hat{d}_{32} \Omega_{3,1}=-\hat{d}_{32} \hat{\sigma}_{1} S_{11}$,

where consideration of the conditions (5.6) and (5.9) allow the appearing elastic modulus,

$\hat{d}_{32}=\frac{1}{3}\left(2 \beta_{4}+\beta_{7}-12 \beta_{5}-6 \beta_{6}\right)$,

to attain either positive or negative values. 
On the other hand, (5.8) yields

$W^{m}=\hat{\beta}_{3} \kappa_{11}^{2}=\hat{\beta}_{3} u_{1,11}^{2}=0$.

As a result, the PDE (4.16) obtains again the form (6.11), with the only difference here being that the single fibre bending stiffness parameter $d^{f}$ is now replaced in (6.10) by $\hat{d}_{32}$.

It is thus seen that, in this pure bending problem, the only simplification/approximation introduced by the bending version of the theory (Section 6.1) confines into a kind of filtering that is applied on the values of the elastic modulus $\hat{d}_{32}$ in a manner that enables only their positive range,

$d^{f}$, to emerge as suitable for use. Again, this conclusion is considerably more specific, as well as simpler, than its counterpart implied in [15].

\section{Conclusions}

The presented, refined hyperelasticity theory of fibre-reinforced materials with fibre-bending stiffness resolves the long-standing indeterminacy problem of the spherical couple-stress, at least as far as this class of fibrous composites is concerned. It thus refines and completes the initial version of the theory [1] without dismissing any of the results obtained, or the conclusions made there. This completed version of the theory achieves its aim by postulating that the couple-stress field is energetically reciprocal to the gradient of some virtual spin field which, unlike the spin field of deformation that is instead employed in Cosserat-type formulations, does not need to be determined.

In the case of fibre-reinforced materials with fibre bending stiffness, this slight modification of the standard postulates of the Cosserat theory is supported by the observation that (i) the fibrespin vector generally differs from the spin vector of the deformation and, in addition, (ii) there is not enough information available to enable precise determination of a relevant dominant spin field. The implied postulate modification thus furnishes the equation of power balance with an additional energy term, which matches the extra energy term that emerges in the strain energy density/function of the earlier formulation $[1,3]$.

This power-energy matching then provides a required extra equation that enables determination the spherical couple-stress outside the conventional equilibrium considerations. As is also detailed in Section 5, the emergence of the implied extra energy term has been clearly noticeable within the small deformation regime [1,3], where the strain energy function is necessarily quadratic in the strains and the curvature-strains. This fact is further discussed and clarified in Section 6, which demonstrates the manner that the spherical couple-stress is determined when a rectangular fibre-reinforced plate is subjected to pure bending within the regime of small deformations.

It is worth noting at this point that the simple application presented in Section 6 reveals that, regardless of whether a relevant boundary value problem deals with small or large elastic deformations, its final solution is essentially completed in two steps. The first of these steps requires determination of the deformation, as well as the symmetric part of the stress and the deviatoric couple-stress, in the light of the relevant formulation detailed in $[1,3]$. The second step then uses the thus obtained initial information and determines the spherical couple-stress in the manner detailed in the present communication. Hence, knowledge of both the deviatoric and the spherical parts of the couple-stress tensor finally enables determination of the antisymmetric part of the stress and, hence, full determination of the non-symmetric stress field.

It is emphasised in this regard that the afore mentioned extra energy term emerges in a natural manner within the quadratic form that the strain energy function necessarily attains in the regime of small elastic deformations. Hence, its appearance in a corresponding strain energy density that may be employed in relevant finite elasticity applications is essentially a physical requirement. 
This is because any admissible form of a strain energy density employed in finite elasticity applications is required to be consistent with its own approximate form that serves the purposes of the linear, infinitesimal deformation version of the theory. In other words, the quadratic form of the strain energy function employed in infinitesimal elasticity applications is necessarily a leadingorder approximation of any admissible form of a strain energy density that may be employed in relevant finite elasticity applications.

In this context, mention should be made, and credit must be given to Ronald Rivlin, who laid foundations for systematic use of invariant theory in the formulation of constitutive equations in continuum mechanics, as well as to later authors who systematically developed invariant theory and worked on construction bases of invariants and tensor functions (e.g., [24]). It is through use of those invariant theory methods that the implied extra energy term emerges in the strain energy function/density of the present hyperelasticity theory and underpins the extra research effort needed towards resolution of the indeterminacy of the spherical part of the couple-stress; a long-standing problem in the couple-stress theory.

\section{Appendix: Solution of the partial differential equation (6.11)}

Solution of the PDE (6.11) on the $x_{1} x_{3}$-plane is achieved with use of the method of characteristics and initially requires a search for the plane curves whose tangent satisfies the equation

$$
\frac{d x_{3}}{d x_{1}}=-\frac{S_{11} x_{1}}{S_{12} x_{3}}
$$

Integration of this separable equation shows that the characteristic lines sought are the ellipses (6.12a)

where the arbitrary function $\lambda\left(x_{2}\right)$ plays the role of an arbitrary integration constant on any $x_{1} x_{3^{-}}$ plane; see also Figure 1, where $\left|x_{1}\right| \leq L_{1},\left|x_{2}\right| \leq h / 2$ and $\left|x_{3}\right| \leq L_{3}$.

Use of (A.1) on any of the implied $x_{1} x_{3}$-planes then transforms the PDE (6.11) into the firstorder ordinary differential equation (ODE)

$$
\frac{d m_{k k}}{d x_{1}}=-\frac{15 S_{11} \hat{m}_{3}}{8 S_{12} x_{3}}=\mp \frac{15 \sqrt{S_{11}} \hat{m}_{3}}{8 S_{12} \sqrt{\lambda^{2}-\left(x_{1}^{2} / S_{12}\right)}},
$$

where (6.12a) is also accounted for. Hence, straightforward integration of this ODE yields (6.12b), whose association with (6.11a) provides the general solution sought for (6.11).

\section{References}

1. Spencer, AJM, Soldatos, KP. Finite deformations of fibre-reinforced elastic solids with fibre bending stiffness. Int J Non-lin Mech 2007; 42: 355-368.

2. Cosserat, E, Cosserat, F. Théorie des Corps Deformables. Hermann: Paris, 1909.

3. Soldatos, KP. Foundation of polar linear elasticity for fibre-reinforced materials. J. Elast. 114, 155178 (2014).

4. Truesdell, C, Toupin, RA. The Classical Field Theories. In: Flugge, S (ed) Encyclopedia of Phyics III/1, Berlin: Springer-Verlag, 1960, 226-793.

5. Mindlin, RD, Tiersten, HF. Effects of couple-stresses in linear elasticity. Arch Ration Mech Anal

6. $1962 ; 11: 415-448$.

7. Koiter. WT. Couple stresses in the theory of elasticity, I and II. Proc. Ned. Akad. Wet. Ser B. 67, 1744 (1964).

8. Mindlin, RD, Eshel, EE. On first strain-gradient theories in linear elasticity. Int. J. Solids Struct. 4, 109-124 (1968). 
9. Eringen, AC. Theory of micropolar elasticity. In: Liebowitz, H. (ed.) Fracture 2, 621-729. Academic Press, New York (1968).

10. Yang, F, Chong, ACM., Lam, DCC, Tong, P. Couple stress based strain gradient theory for elasticity. Int. J. Solids Struct. 39, 2731-2743 (2002).

11.Hadjesfandiari, AR, Dargush, GF. Couple stress theory for solids. Int. J. Solids Struct. 48, 24962510 (2011).

12.Neff, P, Münch, I, Ghiba, I-D, Madeo, A. On some fundamental misunderstandings in the indeterminate couple stress model. A comment on recent papers of A.R. Hadjesfandiari and G.F. Dargush. Int. J. Solids Struct. 81, 233-243 (2016).

13. Münch, I, Neff, P, Madeo, A, Ghiba, I.-D. The modified indeterminate couple stress model: Why Yang et al.'s arguments motivating a symmetric couple stress tensor contain a gap and why the couple stress tensor may be chosen symmetric nevertheless. ZAMM 97, 1524-1554 (2017).

14. Soldatos, KP. On the characterisation of fibrous composites when fibres resist bending - Part III: The spherical part of the couple-stress. Int. J. Solids Struct. 202, 217-225 (2020).

15. Soldatos, KP. Determination of the spherical part of the couple-stress in a polar fibre-reinforced elastic plate subjected to pure bending. Acta Mechanica (doi.org/10.1007/s00707-021-03035-z) (2021).

16. Malvern, LE. Introduction to the Mechanics of a Continuous Medium. Prentice-Hall, Englewood Cliffs, N.J. (1969).

17.Spencer, AJM. Continuum Mechanics. Longman, New York (1980); Dover edn., Mineola, (2004).

18.Soldatos, KP, Shariff, MHBM, Merodio, J. On the constitution of polar fibre-reinforced materials. Mech. Adv. Meter. Struct. (doi: 10.1080/15376494.2020.1729449) (2020).

19.Farhat, AF. Basic Problems of Fibre-reinforced Structural Components when Fibres Resist Bending. PhD Thesis, Sch. Math. Sci., University of Nottingham, Nottingham (2013).

20.Farhat, AF, Soldatos, KP. Cylindrical bending and vibration of polar material laminates. Mech. Adv. Mater. Struct. 22, 885-896 (2015).

21.Soldatos, KP, Aydogdu, M, and Gul, U. Plane strain polar elasticity of fibre reinforced functionally graded materials and structures. J. Mech. Mater. Struct. 14, 497-535 (2019).

22.Aydogdu, M, Aksencer, T, Soldatos, KP. On three-dimensional dynamics of fibre-reinforced functionally graded plates when fibres resist bending. J. Eng. Math. 128, 13 (doi.org/10.1007/s10665021-10128-0) (2021).

23.Soldatos, KP. On the characterisation of fibrous composites when fibres resist bending. Int. J. Solids Struct. 146-149, 35-43 (doi.org/10.1016/j.ijsolstr.2017.10.001) (2018).

24.Spencer, AJM. Ronald Rivlin and invariant theory. Int. J. Eng. Sci. 47, 1066-1078 (2009). 


\section{FIGURES}

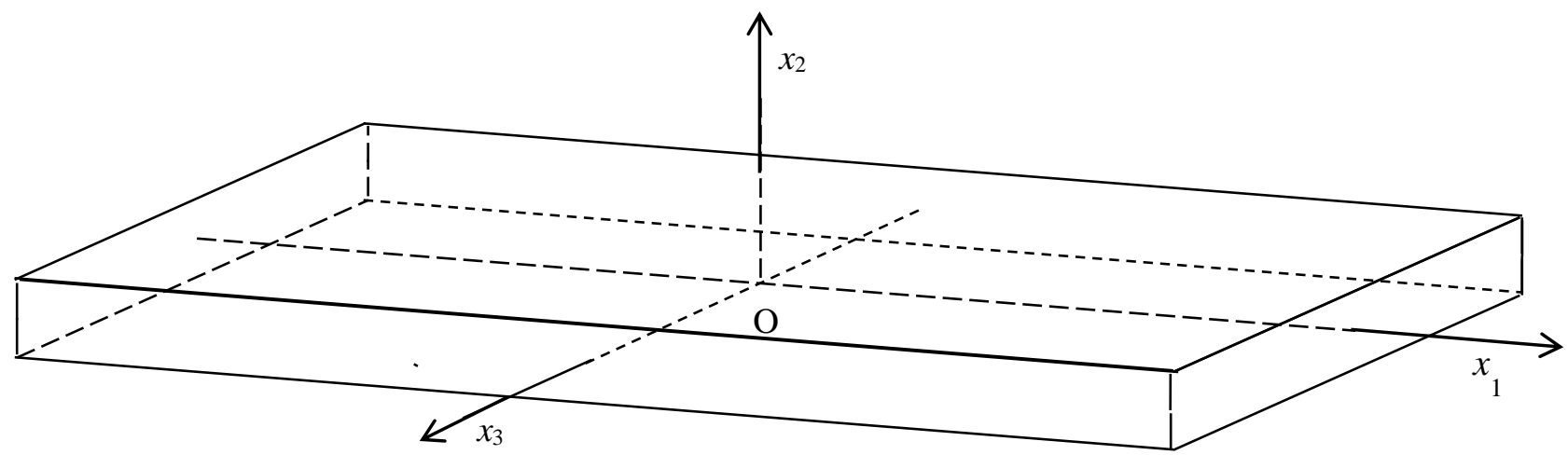

FIGURE 1: Schematic representation of a rectangular plate in a suitably selected Cartesian co-ordinate system, $O x_{i}\left(-L_{1} \leq x_{1} \leq L_{1},-h / 2 \leq x_{2} \leq h / 2,-L_{3} \leq x_{3} \leq L_{3}\right)$. 


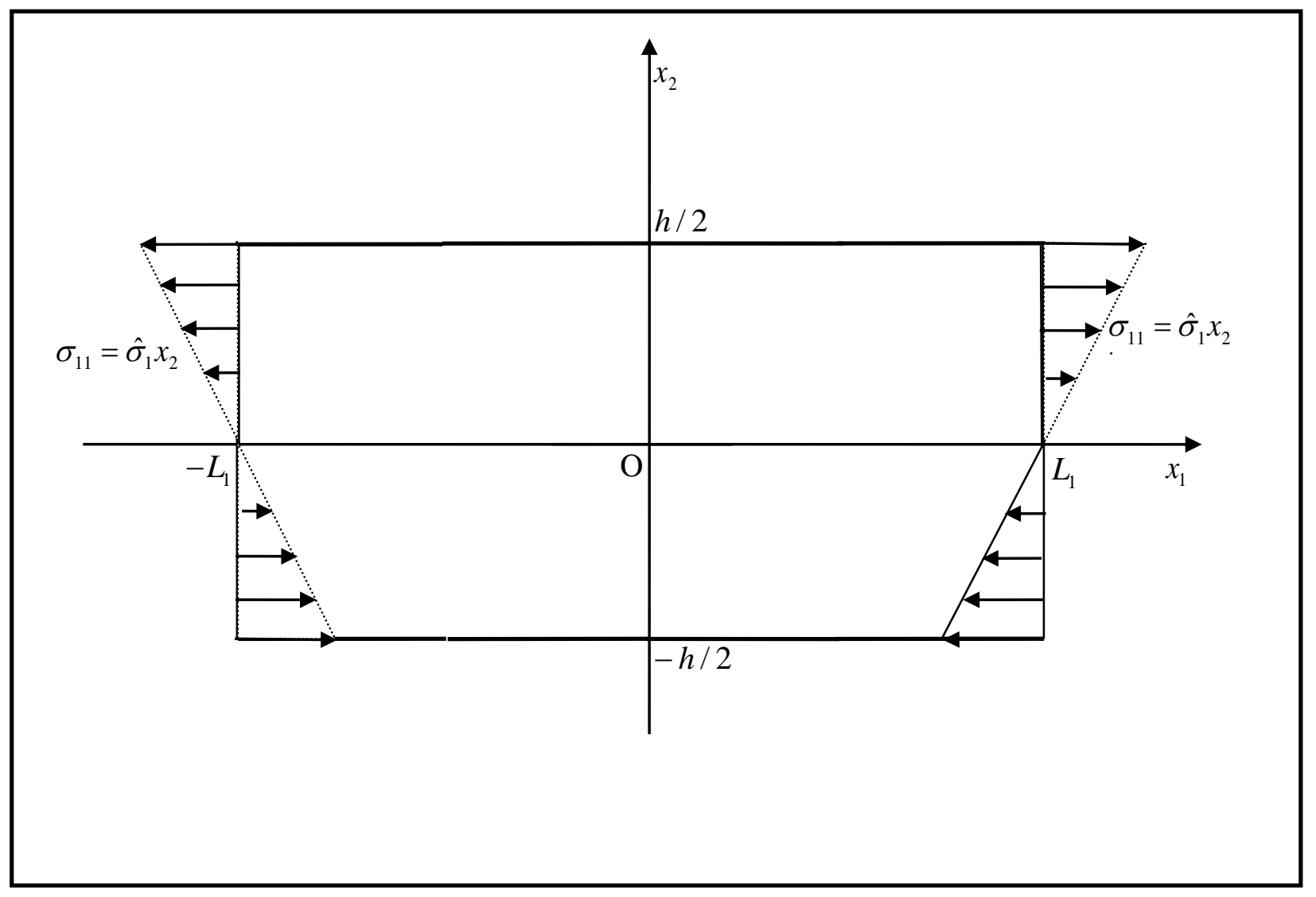

FIGURE 2: Schematic representation of a plate cross-section that is normal to the $x_{3}$-direction, featuring the boundary traction distributions that create pure bending in the case of non-polar linearly elastic material behaviour. 


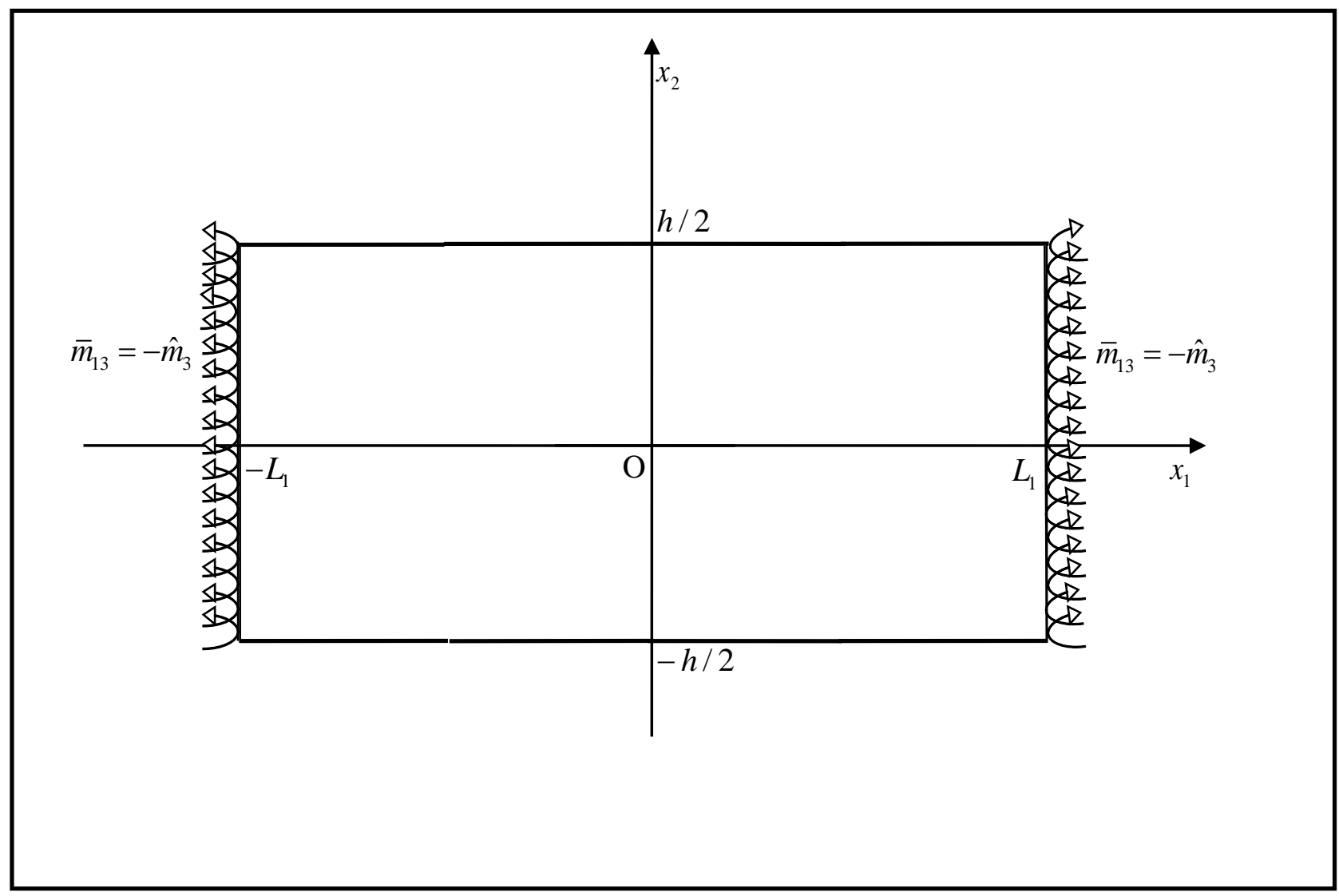

FIGURE 3: Schematic representation of constant couple-traction distributions superposed on the externally applied loading depicted in Figure 2, for a corresponding polar fibre-reinforced elastic rectangular plate to maintain the pure bending deformation (6.3). 